\title{
Originals
}

\section{Type I (Insulin Dependent) Diabetes Mellitus}

\author{
Is There Strong Evidence for a Non-HLA Linked Gene? \\ B. K. Suarez and P. Van Eerdewegh \\ Department of Psychiatry, Washington University School of Medicine, The Jewish Hospital of St. Louis, St. Louis, Missouri, USA
}

\begin{abstract}
Summary. A recent investigation of families containing two and three consecutive generations affectec with Type I (insulin dependent) diabetes mellitus has led to speculation that there is a second susceptibility gene, not linked to the major histocompatibility complex. These families differ in important respects from families which have provided the strongest evidence for HLA linkage, namely, families with two or more affected siblings. Computer simulations were designed to test the hypothesis that these two types of families were drawn from the same population of insulin dependent patients. The results indicate that this hypothesis is tenable and that the consecutive generation families probably failed to yield strong evidence for an HLA linked susceptibility gene because of ascertainment bias combined with probable incorrect specifications of the mode of inheritance of insulin dependent diabetes.
\end{abstract}

Key words: Inheritance, insulin dependent diabetes, HLA complex, genetic linkage, ascertainment bias, family studies, computer simulations.

During the last decade it has become clear that diabetes mellitus can be subdivided into at least two independent disorders. Type $I$ is characterized by early onset, dependency on exogenous insulin and a pronounced HLA association [1]. Type II is characterized by later onset, no exogenous insulin dependence and no HLA association. There is also a growing suspicion that Type I diabetes itself may be further subdivided into two aetiological disorders that share in common an early onset, insulin dependency and HLA associations [2, 3]. There appears to be no agreement, however, whether the Type I diabetes associated with HLA-Dw3-B8 bearing haplotypes is really any different from that associated with HLA-Dw4-B15 haplotypes [4-6].
Recently, Barbosa et al. [7] have argued that there may be yet another type of Type I diabetes (IDDM) which is not linked to the HLA complex. Their claim is based on the analysis of two series of multiplex families. The first series, designated the MS families (multiple affected siblings), were ". . . ascertained to maximize the likelihood of dealing with a genetically homogenous form (or forms) of autosomal recessive IDDM" [7, p 597]. The second series of families, designated the $\mathrm{MG}$ families (multiple affected generations), were ascertained ". . . to maximize the likelihood of obtaining a homogenous type of disease within a family, on an autosomal dominant mode of inheritance" [7, p 592]. Evidence for their claim that the MS families are segregating an HLA linked form of the disease while the MG families are not can be summarized by the following findings. When analysed for HLA linkage the 21 MS families gave positive linkage results when Type I diabetes was treated as a recessive disorder with either partial or full penetrance [8]. By contrast, when the $28 \mathrm{MG}$ families were analysed for HLA linkage by modelling Type I diabetes as dominantly transmitted, no compelling evidence was obtained, although as the penetrance of the susceptibility genotypes were allowed to decrease the evidence for linkage improved. Finally, when these same 28 MG families were reanalysed by supposing that Type I diabetes was recessively transmitted, they again failed to yield any compelling evidence for linkage.

On the basis of these observations Barbosa et al. [7] suggest that the difference between the MS and the MG families is ". . . compatible with a genetic difference between these two types of families and seems to confirm the theory of genetic heterogeneity in IDDM."

It is obvious that if there is a subset of Type I diabetic families segregating a dominantly transmitted form of the disorder, the sample of MG families obtained by Barbosa et al. [7] are more likely to con- 
tain them than a randomly ascertained sample. However, in these data the ascertainment procedure through which the MG families were obtained precludes a test of this hypothesis.

We report here the results of a series of computer simulations designed to test the hypothesis that both the MS and MG families could have been drawn from the same homogenous Type I diabetic population. These simulations suggest that the inconsistent results obtained by Barbosa et al. [7] could be due to incorrect specification of mode of inheritance combined with ascertainment bias rather than due to genetic heterogeneity.

\section{Methods}

\section{Family Structure}

The structures of the $28 \mathrm{MG}$ families reported by Barbosa et al. [7] are quite variable ranging in size from a four member - two generation family, to a 30 member - four generation family. Rather than

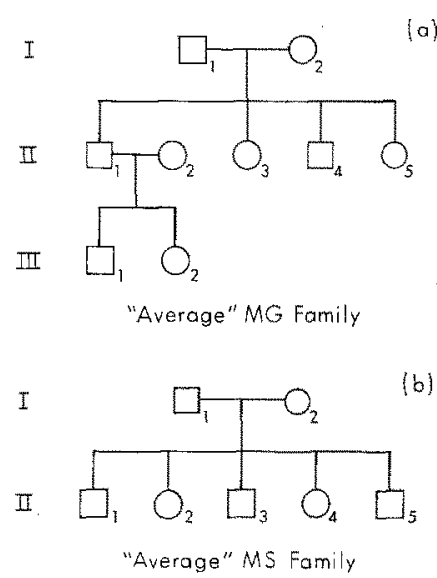

Fig. 1. a The "average" multiple affected generation (MG) family structure and b the "average" multiple affected sibling (MS) family structure used in the simulations try to duplicate each family structure in our simulations, we elected to settle on an "average family" (Fig. 1a).

\section{Transmission Models}

Since the genetics of Type I diabetes is unknown, it was important to select a range of transmission models which are parsimonious on other grounds. That is, the model should not only predict the prevalence of the disorder in the general population, but also the disorder's incidence in the relatives of probands. Unfortunately, estimates of the incidence of Type I diabetes in relatives vary greatly. Accordingly, we have selected five parameter sets that span the reported izcidence rates. For the simulations reported here, the transmission model used assumes that Type I diabetes is determined by a two allele locus which gives rise to the phenotypes "affected" and "unaffected". The model contains four parameters, the gene frequency, $q$, of the "-." Type 1 diabetes susceptibility allele $(p=1-q$, is the frequency of its allelomorph, " + "), and three penetrances denoted by $f_{1}, f_{2}$ and $f_{3}$ corresponding to the respective genotypes,+++- and -- . The parameters are chosen such that $f_{1} p^{2}+2 f_{2} p q+f_{3} q^{2}$ is the population prevalence of Type I diabetes which is assumed to be $1.6 \times 10^{-3}$ to make the present analysis comparable to that reported earlier [9].

The five parameter sets used to model the transmission of Type I diabetes in the simulated families were chosen such that the resulting concordance of monozygotic co-twins and the full siblings of probands - the two best studied classes - cover the range of reported rates $[1,10-16]$. Table 1 lists the values of the parameters used and the resulting incidence rates in monozygotic co-twins and full siblings of probands. The distribution of these five transmission parameter sets with respect to the underlying additive and dominance variance components of the generalized single locus model is shown in Figure $2[26,27]$.

\section{Simulations}

For each Type I diabetic transmission model listed in Table 1 (models A-E), families with the structure shown in Figure la were simulated by Monte Carlo methods until 28 families meeting the ascertainment criterion (that is, multiple affected generations) were obtained. Since for practical purposes the HLA complex is completely informative with respect to linkage, we assigned the HLA haplotypes in the simulations such that each parent had unique haplotypes. The probability of a crossover between the HI A marker and the Type I diabetic locus was determined for each of the five transmission models by selecting the recombina-

Table 1. Parameter sets $\left(\mathrm{q}, \mathrm{f}_{1}, \mathrm{f}_{2}\right.$ and $\left.\mathrm{f}_{3}\right)$ of models $\mathrm{A}-\mathrm{E}$ used to generate families for the simulations. Each parameter set is consistent with a population prevalence of $0.16 \%$ for Type I diabetes. Each parameter set will give rise to a different incidence of Type I diabetes in relatives of a proband. See [9] for further details

\begin{tabular}{|c|c|c|c|c|c|}
\hline \multirow[t]{2}{*}{ Parameters } & \multicolumn{5}{|c|}{ Generating sets } \\
\hline & A & B & $\mathrm{C}$ & $\mathrm{D}$ & $\mathrm{E}$ \\
\hline Gene frequency, q & 0.041112 & 0.045781 & 0.04182 & 0.019118 & 0.009220 \\
\hline Penetrance of ++ genotype & 0.000115 & 0.000160 & 0.00049 & 0.000110 & 0.000052 \\
\hline Penetrance of +- genotype & 0.004377 & 0.005406 & 0.00475 & 0.031877 & 0.080468 \\
\hline Penetrance of - - genotype & 0.679777 & 0.468472 & 0.44123 & 0.817811 & 0.935217 \\
\hline Recombination fraction & 0.146 & 0.139 & 0.139 & 0.112 & 0.047 \\
\hline \multicolumn{6}{|l|}{ Incidence of Type I diabetes in: } \\
\hline Monozygotic twins & $49 \%$ & $29 \%$ & $21 \%$ & $18 \%$ & $12 \%$ \\
\hline Full sibs & $14 \%$ & $8 \%$ & $6 \%$ & $6 \%$ & $5 \%$ \\
\hline
\end{tabular}


Table 2. Transmission models used to analyse the families simulated under models $\mathrm{A}-\mathrm{E}$. Model $\mathrm{G}$ is the correct family generating model and changes according to which generating model is used

\begin{tabular}{llllllll}
\hline Parameters & \multicolumn{7}{l}{ Models used to analyse families for linkage } \\
\cline { 2 - 8 } & $\mathrm{G}$ & 1 & 3 & 5 & 7 & 9 & $\mathrm{R}$ \\
\hline Gene frequency & See Table 1 & 0.008032 & 0.002760 & 0.001601 & 0.001144 & 0.000889 & 0.056569 \\
Penetrance of ++ genotype & for correct & 0 & 0 & 0 & 0 & 0 & 0 \\
Penetrance of + - genotype & generating & 0.1 & 0.3 & 0.5 & 0.7 & 0.9 & 0 \\
Penetrance of -- genotype & model & 0.1 & 0.3 & 0.5 & 0.7 & 0.9 & 0.5 \\
\hline
\end{tabular}

tion fraction that minimised the $\mathrm{X}^{2}$ goodness-of-fit to published affected sib pair data $[9,17,18]$.

The phenotype of person II-2, who marries into the family, was ignored in ascertaining the pedigrees. For each of the five transmission models in Table 1 the simultion of the $28 \mathrm{MG}$ families was repeated 10 times.

\section{Linkage Analysis}

Each of the ten replicates of the $28 \mathrm{MG}$ families simulated under each of the five generating parameter sets was analysed for linkage under seven competing models (Table 2). These include the correct "generating model" (G), five incompletely penetrant dominant models, and an incompletely penetrant recessive model (R). The incompletely penetrant dominant models are designated models $1,3,5,7$ and 9 , corresponding to penetrances, that is, $f_{2}$ and $f_{3}$, equal to $0.1,0.3,0.5,0.7$ and 0.9 , respectively. For model $\mathbf{R}$ the penetrance vector is $0,0,1 / 2$ for $f_{1}, f_{2}$ and $f_{3}$, respectively. In order to assure that the population prevalence remained constant for each "dominant" model, the gene frequency was fixed at $\mathrm{q}=1$ $\sqrt{(1-K / F)}$ where $K$, the population prevalence, is 0.0016 and $F$ is the penetrance under the particular model 1-9. For the incompletely penetrant recessive model the gene frequency was fixed at $q$ $=\sqrt{\mathrm{K} / \mathrm{F}}$.

All analyses were performed with the computer program LIPED [19] using the lod score method. The lod score is the logarithm to the base 10 of the ratio of the likelihood of the sample (uncorrected for ascertainment) evaluated at some $\theta$ and the likelihood of the sample evaluated at a recombination fraction of $\theta=1 / 2$ (that is, under the hypothesis of no linkage). In a random sample $\hat{\theta}$, the value of $\theta$ which gives the maximum lod score, is the recombination fraction that maximises the probability of obtaining the observed sample. A lod score of 3 , for instance, means that the hypothesis of linkage is $10^{3}$ times more likely than the hypothesis of no linkage. By convention, a lod score of 3 is taken as confirmation of linkage. Lod scores are sometimes referred to as Z-scores and we use both terms interchangeably.

\section{Results}

\section{Analysis of Linkage in Families with Multiple Generations Affected}

The lod score distribution obtained from each of the seven models used to analyse the data are reported in Figure 3 for the five generating parameter sets A-E. Each curve was obtained by averaging over the 10 replicates of the $28 \mathrm{MG}$ families. Taking the mean lod score curve obtained by analysing the families with the correct generating parameter set as a stand-

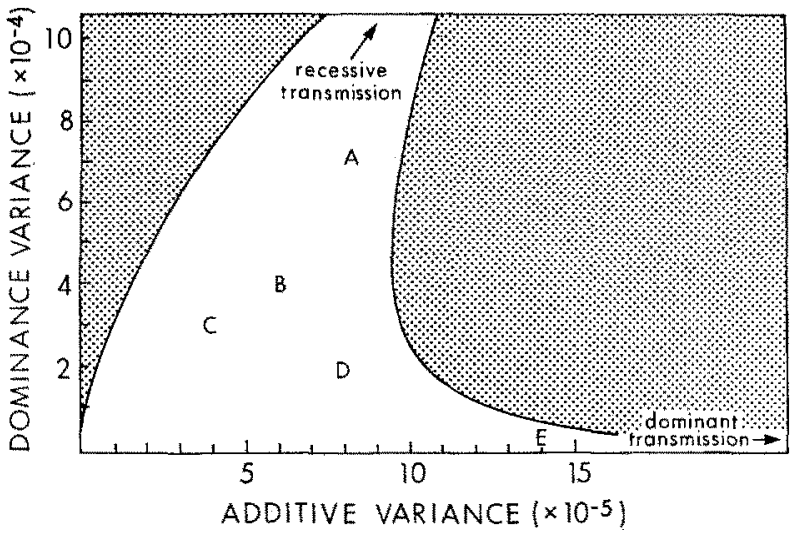

Fig. 2. Distribution of the five parameter sets used to generate the MG families. The stippled region represents combinations of the additive and dominance variances that lie outside of the incompletely penetrant single gene model $[9,26]$ for further details

ard against which to judge the other models, Figure 3 shows that models $1-9$ consistently give poor results in the sense that they peak at a lower lod score and uniformly yield estimates of the recombination fraction that are too large. Model R, by contrast, gives results virtually identical to the "correct" lod curves for parameter sets $\mathrm{A}$ and $\mathrm{B}$. This is not too suprising since the penetrance vectors used to generate the $A$ and $B$ families are similar to the penetrance vector of model R. Data sets C, D and E, however, are progressively more "dominant" and for each of these model $\mathrm{R}$ becomes less adequate (Figs. 2 and 3 ).

\section{Analysis of Linkage in Families with Multiple Affected Siblings}

One of the observations that led Barbosa et al. [7] to suggest that their MG families may have a different type of Type I diabetes than their MS families was the finding that, when analysed with model $R$, the MS families yielded strong evidence for HLA linkage whereas the MG families did not. Accordingly, we carried out one further set of simulations to determine if one of the generating parameter sets listed in Table 1 could produce results similar to those reported by Barbosa et al. [8]. "Average MS families" 


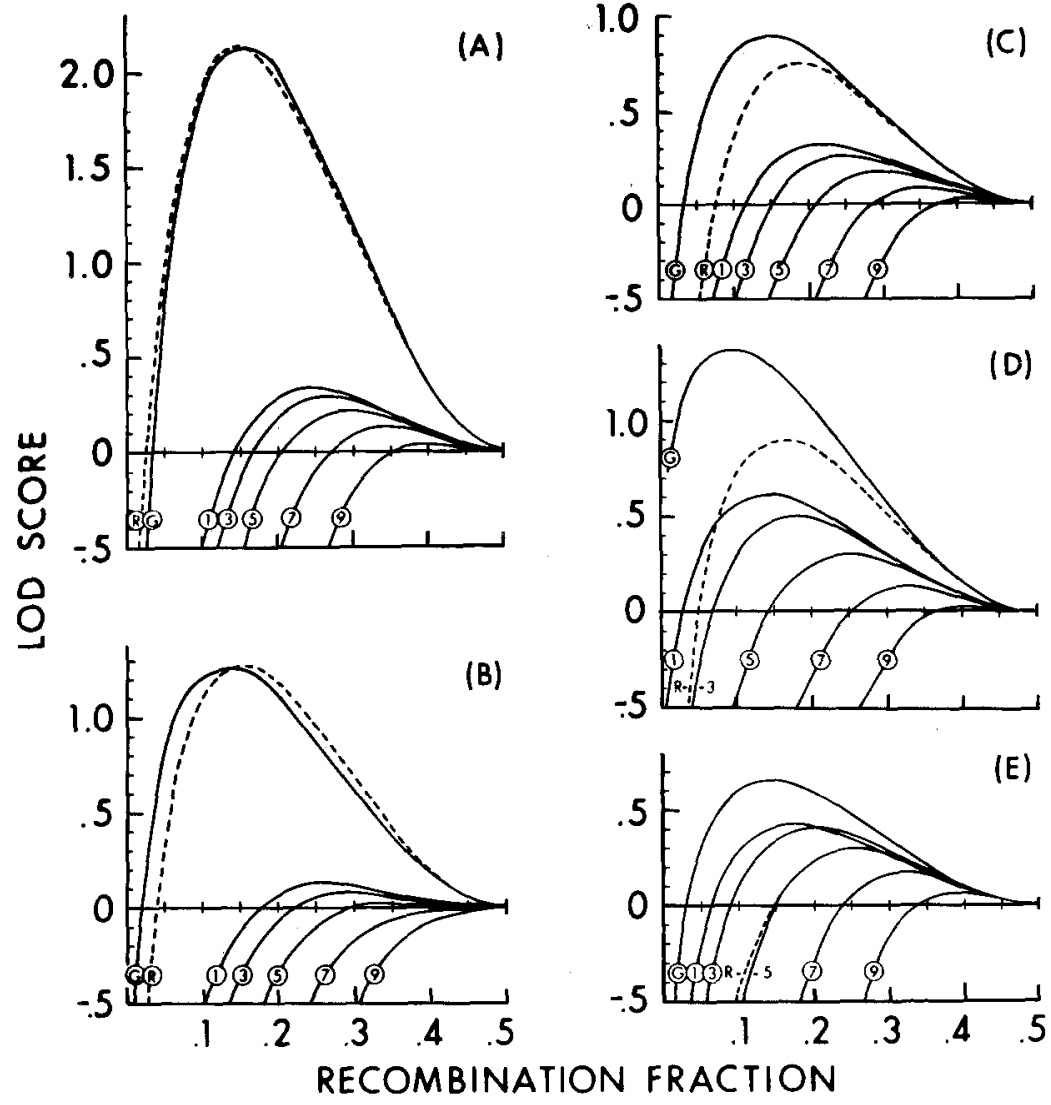

Fig. 3. Each mean lod score curve was obtained by simulating $\mathrm{MG}$ families with parameter sets A to $E$ and then analysing them with models $G$ (the correct model), $\mathrm{R}$ (the incompletely penetrant recessive model - dashed curve), and 1-9 (the five incompletely penetrant dominant models). The lod score is the exponent of 10 which gives the likelihood of the hypothesis of linkage (at some $\theta$ ) compared to the hypothesis of no linkage (that is, $\theta=1 / 2$ ). The curves were drawn by summing over all $280 \mathrm{MG}$ families generated with a particular parameter set and dividing by 10 (the number of replicates) to obtain the mean curve that could be expected from a study of 28 such families
(Fig. 1b) were simulated as described above except that now the ascertainment rule required that neither parent be affected while requiring a minimum of two offspring to be affected. Since the results of the linkage analysis with models $1-9$ and $R$ generated under parameter set $\mathrm{E}$ best approximated the findings reported by Barbosa et al. [7] for their MG families we decided to simulate our MS families using parameter set $\mathrm{E}$. After 21 families meeting the ascertainment criteria were generated, linkage analysis was carried out under three models, two used earlier ( $G$ and $R$ ), and a fully recessive model with a gene frequency of $\mathrm{q}=0.04$ which preserves the proper population prevalence. These simulations were repeated 20 times. Figure 4 displays the distribution of the maximum lod scores plotted against their $\hat{\theta}$ 's for each of the 20 replicates. The mean maximum lods were found to be $2.91(\bar{\theta}=0.05), 2.69(\bar{\theta}=0.063)$ and $2.49(\bar{\theta}=0.194)$ for $G, R$ and the fully penetrant recessive model, respectively.

\section{Discussion}

The simulations described above were designed to test the hypothesis that both the MS and the MG families could have been drawn from the same
Type I diabetic population. In particular, we wanted to evaluate the possibility that the disparity in the linkage results from these two types of families could have been due to the methods through which they were ascertained combined with possible incorrect assignment of the mode of inheritance.

Since we treated $\theta$ as the only unknown parameter, the maximum lod score from each replicate gives the maximum likelihood estimate of the true recombination fraction - had the families been randomly sampled. Maximum likelihood estimates are, in general, asymptotically unbiased. The families were not, however, randomly sampled and as a result it is difficult to determine if the bias is principally due to the ascertainment procedure or to the size of the sample. Any errors due to ascertainment bias, however, are compounded if the parameters defining the mode of inheritance are incorrectly specified. Reference to Figure 3 suggests the following three conclusions:

(1) Even when the correct mode of inheritance is known (model G), $28 \mathrm{MG}$ families with the structure shown in Figure 1a generally will be insufficient to confirm linkage since none of the maximum mean lod scores $(\bar{\theta}$ 's $)$ are greater than 3.0. The generating model that is closest to Mendelian dominant transmission, model $\mathrm{E}$, gave the lowest mean $\mathrm{Z}$-score so that if Type I diabetes was transmitted in a similar 


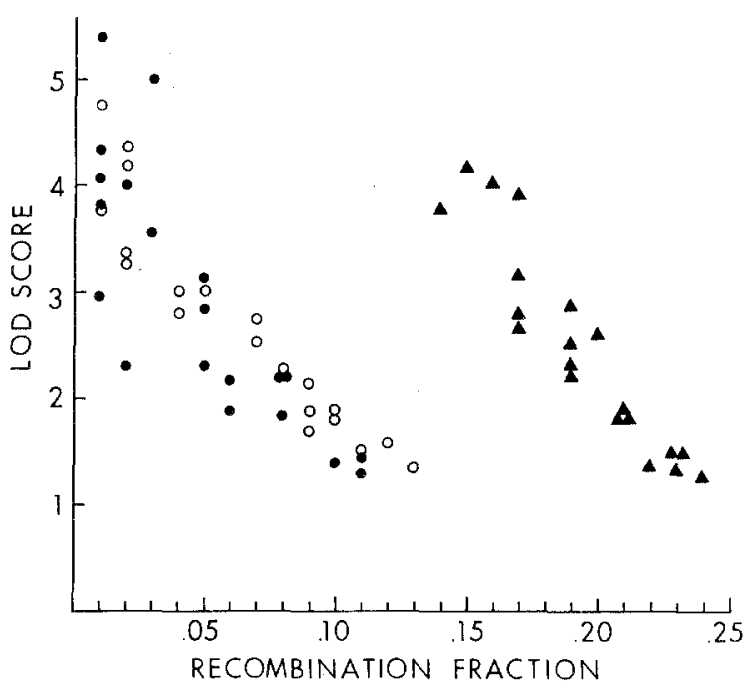

Fig. 4. Maximum lod scores and their associated $\hat{\theta}$ 's for each of the 20 replicates of 21 MS families generated with parameter set $\mathrm{E}$ and anlysed by treating them under the correct model $(\bullet)$, under an incompletely penetrant recessive model $(O)$ and under a fully penetrant recessive model $(\boldsymbol{A})$

fashion, more than $125 \mathrm{MG}$ families would be required, on average, to confirm the linkage.

(2) When the data are analysed with the (incorrect) incompletely penetrant dominant models $1-9$, the resulting Z-score distributions are qualitatively similar to those reported by Barbosa et al. [7]. That is, in every case as $f_{2}=f_{3} \rightarrow 0$, the lod score increases and, as expected, $\hat{\theta}$ becomes smaller. Even under the "best" model, that is, when $\mathrm{f}_{2}=\mathrm{f}_{3}=0.1$, the maximum lod score remains small, however.

(3) When the data are analysed with the (incorrect) incompletely penetrant recessive model $R$, mean lod scores are obtained which for two generating sets, A and B, attain a larger maximum lod score than obtained under the correct models. Indeed, with the exception of parameter set $\mathrm{E}$, model $\mathrm{R}$ presents stronger evidence for linkage than models 1-9 despite the fact that the families were ascertained to appear "dominant". The fitting of model $R$ to the families generated under parameter set $\mathrm{E}$ gave results similar to those reported by Barbosa et al. [7].

When parameter set $\mathrm{E}$ is used to generate MS families, which in terms of their configuration appear "recessive", the evidence for HLA linkage is considerably stronger than obtained from the larger number of MG families. It is instructive to note, however, that the maximum mean lod score and its corresponding $\bar{\theta}$, for the divergent models $G(\operatorname{read} E)$ and $R$, give essentially identical results. This finding suggests that while families with multiple affected siblings may be informative for detecting linkage, they may provide little discrimination between com- peting models of transmission. This may explain why some investigators have concluded that susceptibility to Type I diabetes is dominant $[21,22]$ while others have concluded that it is recessive $[23,24]$.

There are a number of specific differences between the types of families we have simulated and those reported by Barbosa et al. [7] and these differences should be considered when interpreting our results. Firstly, in their analysis Barbosa et al. [7] assumed that the population prevalence of Type I diabetes is $0.189 \%$ [25], whereas we chose to generate families under a population prevalence of $0.16 \%$ so that results could be directly compared to an earlier analysis [9]. In terms of the incorrect models used to analyse the linkage data, the above difference in assumed population prevalence gives rise to a negligible difference in the gene frequency. Secondly, our simulation did not allow for variable age of onset. That is, our simulations assume that all persons are through their risk period so any unaffected persons will remain unaffected. In their study, however, there are many individuals who have not yet passed through the risk period and some of these can reasonably be expected to become affected in the future. Indeed, approximately $50 \%$ of the unaffected persons in the MG families of Barbosa et al. [7] have yet to complete their third decade.

Perhaps the most important difference between our simulations and the real data is that we elected to generate our MG and MS families under an "average" fixed structure. While this decision simplified the simulations, it is probably the case that large kindreds carry proportionally greater information for detecting linkage than do smaller families even when the total number of persons is held constant.

In the original description of their MS families Barbosa et al. [20] conjectured that the transmission of Type I diabetes is quasi-dominant. They offered a diathesis-stress model that posits a single Type I diabetes susceptibility allele which, in heterozygotes, gives rise to the disorder only in the presence of sufficient environmental stimuli. Homozygotes for the susceptibility gene, however, were hypothesized to display Type I diabetes in the presence of a much lower environmental loading. In terms of the generalized single locus model used here, their original conjecture would translate into a penetrance vector similar to that of parameter set $\mathrm{E}$. That is, a very low $\mathrm{f}_{1}$ accounting for sporadic cases, an $\mathrm{f}_{2}$ which, while low, nonetheless accounts for most of the Type $I$ diabetic cases, and an $f_{3}$ which approaches $100 \%$ but only accounts for a small percentage of patients due to the low frequency of the susceptibility gene. It is interesting to note that with respect to linkage analysis of just affected sibling pairs, parame- 
ter sets in the vicinity of point $\mathrm{E}$ give the lowest goodness-of-fit $\mathrm{X}^{2}$ [9].

The remarkably close agreement between the results obtained from parameter set $\mathrm{E}$ and those reported by Barbosa et al. [7] should not be interpreted to mean that parameter set E is "correct". Indeed, we suspect that any parameter set in the vicinity of $E$ would yield similar results. What the simulations do suggest, however, is that the linkage results obtained by Barbosa et al with the MS families [8] and their MG families [7] are compatible with the hypothesis that the insulin dependent diabetes mellitus in these families is determined by the same incompletely penetrant locus linked to the HLA complex. These findings need not be interpreted as proving that there are no non-HLA linked Type 1 diabetes susceptibility genes. They only suggest that the MG families, as ascertained and analysed, fail to provide the needed confirmation.

Acknowledgements. We are grateful to Mr. J. Mullaney for his skillful programming assistance and to Drs. T. Reich, J. Rice, and C. R. Cloninger for their valuable suggestions. This work was supported in part by USPHS grants MH-31302, MH-14677 and GM28067.

\section{References}

1. Cudworth AG (1978) Type I diabetes mellitus. Diabetologia 14: 281-291

2. Hodge SE, Rotter JI, Lange KL (1980) A three-allele model for heterogeneity of juvenile onset insulin dependent diabetes. Ann Hum Genet 43: 399-412

3. Rotter JI, Hodge SE (1980) Racial differences in juvenile-type diabetes are consistent with more than one mode of inheritance. Diabetes 29: 115-118

4. Bengsch N, Köbberling J (1979) No evidence for two HLAassociated genes. In: WaldhäusI N, Alberti KGMM (eds) 10th Congress of the International Diabetes Federation. Excerpta Medica, Amsterdam, p 19

5. Watson B, Sachs J, Jaraquamada D, Festenstein H (1979) Type I diabetes and the D-locus. In: Waldhäusl W, Alberti KGMM (eds) 10th Congress of the International Diabetes Federation. Excerpta Medica, Amsterdam, p 249-250

6. MacDonald MJ (1980) The frequencies of juvenile diabetes in American blacks and caucasians are consistent with dominant inheritance. Diabetes 19: 110-114

7. Barbosa J, Chern MM, Anderson VE, Noreen H, Johnson S, Reinsmoen N, McCarty R, King R, Greenberg L (1980) Linkage analysis between the major histocompatibility system and insulin-dependent diabetes in families with patients in two consecutive generations. J Clin Invest 65: 592-601

8. Barbosa J, Chern MM, Noreen H, Anderson VE, Yunis EJ (1978) Analysis of linkage between the major histocompatibility system and juvenile, insulin-dependent diabetes in multiplex families. J Clin Invest 62: 492-495

9. Suarez BK, Hodge SE, Reich T (1979) Is juvenile diabetes determined by a single gene closely linked to HLA? Diabetes 28: $527-532$

10. Harris $\mathbf{H}$ (1950) The familial distribution of diabetes mellitus:
A study of the relatives of 1241 diabetic propositi. Ann Eugen 15: $95-110$

11. Simpson N (1964) Multifactorial inheritance. A possible hypothesis for diabetes. Diabetes 13: 462-471

12. Gottlieb M, Root H (1968) Diabetes mellitus in twins. Diabetes 17: 693-704

13. Tattersall RB, Fajans SS (1975) A difference between the inheritance of classical juvenile-onset and maturity onset diabetes of young people. Diabetes 24: 44-53

14. Nelson PG, Pyke DA, Cudworth AG, Woodrow JC, Batchelor JR (1975) Histocompatibility antigens in diabetic identical twins. Lancet II: 193-194

15. Nerup J, Platz P, Ortved Andersen O, Christy M, Egeberg J, Lyngsøe J, Poulsen JE, Ryder LP, Thomsen M, Svejgaard A (1976) HLA, autoimmunity and insulin-dependent diabetes. In: Creutzfeldt W, Köbberling J, Neel JV (eds) The genetics of diabetes mellitus. Springer, Berlin Heidelberg New York, p. 106-114

16. Barbosa J, Chern M, Greenberg L (1978) Segregation analysis of juvenile-onset, insulin-dependent diabetes (JIDD) in 136 families. Diabetes 27 [Suppl 2]: 458

17. Suarez BK (1978) The affected sib pair IBD distribution for HLA-linked disease susceptibility genes. Tissue Antigens 12: 87-93

18. Suarez BK, Rice J, Reich T (1978) The generalized sib pair IBD distribution: Its use in the detection of linkage. Ann Hum Genet 42: 87-94

19. Ott J (1974) Estimation of the recombination fraction in human pedigrees: Efficient computation of the likelihood for human linkage studies. Am J Hum Genet 26: 588-597

20. Barbosa J, King R, Noreen H, Yunis EJ (1977) The histocompatiblity system in juvenile, insulin-dependent diabetic multiplex kindreds. J Clin Invest 60: 989-998

21. Spielman RS, Baker L, Zmijewski CM (1979) Inheritance of susceptibility to juvenile onset diabetes. In: Sing C, Skolnick M (eds) Genetic analysis of common diseases: Applications to predictive factors in coronary heart disease. Alan R Liss, New York, p 567-585

22. Svejgaard A, Platz P, Ryder LP, Staub Nielson L, Thomsen $M$ (1975) HLA and disease associations - a survey. Transplant Rev 22: 3-34

23 Rubinstein P, Suciu-Foca N, Nicholson JF (1977) Genetics of juvenile diabetes mellitus. A recessive gene closely linked to HLA D and with 50 per cent penetrance. N Engl J Med 297: 1036-1040

24. Thomson G, Bodmer W (1977) The genetic analysis of HLA and disease associations. In: Dausset J, Svejgaard A (eds) HLA and disease. Williams \& Wilkins, Baltimore, p 84-93

25. Kyllo C, Nuttall F (1978) Prevalence of diabetes in school age children in Minnesota. Diabetes 27: 57-60

26. Suarez BK, Reich T, Trost J (1976) Limits of the general twoallele single locus model with incomplete penetrance. Ann Hum Genet 40: 231-244

27. Suarez BK, Fishman PM, Reich T (1977) Estimating the parameters of the incompletely penetrant single locus model using multiple populations. Hum Hered 27: 336-351

Received: 24 April 1980,

and in revised form: 17 November 1980

Dr. B. K. Suarez

Department of Psychiatry

Washington University School of Medicine

The Jewish Hospital of St. Louis

St. Louis, MO 63110

USA 